\title{
A Flexible Multifunctional Sensor Based on Carbon Nanotube/Polyurethane Composite
}

\author{
P. Slobodian, P. Riha, R. Benlikaya, P. Svoboda and D. Petras
}

\begin{abstract}
A sensor was made of a polymer composite composed of electrically-conductive carbon nanotubes embedded in elastic polyurethane. The composite was prepared by using a polyurethane filter membrane, enmeshing it and melding together with carbon nanotubes. Testing has shown that the composite can be elongated as much as $400 \%$ during which the electrical resistance is increased 270 times. The composite is also sensitive to compression and to organic solvent vapors. These properties indicate the composite could have applications as a highlydeformable strain and chemical vapors sensing element and also as flexible electromagnetic shielding or protection against lightning. As an example of the use of the composite as a strain sensor, the pressure variation between a shoe and floor during walking and knee flexion during cycling have been monitored.
\end{abstract}

Index Terms - Carbon Nanotubes, Chemical Vapor Sensing, Deformation Sensing, Polymer Composite.

\section{INTRODUCTION}

$\mathrm{T}$ HE sensitivity of a strain gauge is usually measured by a gauge factor $(G F)$ which is defined as the relative electrical resistance change divided by the applied strain, $G F=\left(\Delta R / R_{0}\right) / \varepsilon$. To have a high sensitivity, that is, a high change in resistance for the same strain, a higher value of gauge factor is desirable. The copper has a $G F$ of about 2.5, the metal sheet strain gauges have values of $G F 2-5, G F$ of the pure MWCNT network (buckypaper) was experimentally determined as 0.34 [1], or 7 [2] at small strain about $0.1 \%$ due to the weak van der Waals attraction between nanotubes. $G F$ of stretchable composite consisting of multi-walled carbon

Manuscript received December 11, 2012. This work was supported by the Operational Program of Research and Development for Innovations cofunded by the European Regional Development Fund (ERDF), the National budget of the Czech Republic within the framework of the Centre of Polymer Systems project (Reg. No.: CZ.1.05/2.1.00/03.0111). This article was also supported by the Fund of Institute of Hydrodynamics AV0Z20600510.

P.S., D.P., R.B., P.S. Authors are with the Polymer Centre, Faculty of Technology, T. Bata University, T.G.M. 275, 76272 Zlin, Czech Republic and the Centre of Polymer Systems, University Institute, T. Bata University, Nad Ovcirnou 3685, 76001 Zlin, Czech Republic. R.B is also with Balikesir University, Faculty of Necatibey Education, Department of Secondary Science and Mathematics Education, 10100, Balikesir, Turkey. (P.S. corresponding author, phone: +420576031350 ; fax: +420576031444 ; e-mail: slobodian@ft.utb.cz); (e-mail:ruhan@balikesir.edu.tr; svoboda@ft.utb.cz; petras.david@seznam.cz).

P. R. Author is with the Institute of Hydrodynamics, Academy of Sciences, Pod Patankou 30/5, 16612 Prague 6, Czech Republic (e-mail: riha@ih.cas.cz). nanotubes network/polyurethane (MWCNT/PU) stretched up to $400 \%$ and $G F$ was found to be nearly 69 at this strain [3].

The purpose of this paper is to introduce sensing properties of novel MWCNT/PU composite when subjected not only to elongation but also to bending and compression and also to the exposure to organic solvents of different polarities at the same vapor pressure. Similar concepts for such composite structures are used as novel types of sensors [4] or as an active part of sensing composites $[5,6]$.

\section{EXPERIMENTAL}

Two different types of MWCNT were used for the composite preparation. The purified MWCNTs produced by chemical vapor deposition of acetylene, were supplied by Sun Nanotech Co. Ltd., China. According to the supplier, the nanotube diameter is $10-30 \mathrm{~nm}$, length $1-10 \mu \mathrm{m}, \sim 90 \%$ purity and a resistivity of $0.12 \Omega \mathrm{cm}$ [7]. The other MWCNTs were Baytubes C70 denoted here MWCNTs (BT), produced by chemical vapor deposition and supplied by BayerMaterial Science AG, Germany (diameter 5-20 nm, length 1-10 $\mu \mathrm{m}$, Cpurity > $99 \%$ and bulk density $140-230 \mathrm{~kg} / \mathrm{m}^{3}$ declared by supplier). The oxidized MWCNTs were prepared in a glass reactor filled with $300 \mathrm{~cm}^{3}$ of $0.5 \mathrm{M} \mathrm{H}_{2} \mathrm{SO}_{4}, 1.5 \mathrm{~g}$ of $\mathrm{KMnO}_{4}$ and $0.5 \mathrm{~g}$ of MWCNTs. The dispersion was sonicated at $40^{\circ} \mathrm{C}$ for an hour using UP-400S Dr. Hielsher GmbH apparatus (horn $\mathrm{H} 7$, amplitude $88 \mu \mathrm{m}$, power density $300 \mathrm{~W} / \mathrm{cm}^{3}$, frequency $24 \mathrm{kHz}$ ) at $50 \%$ power of the apparatus and in $50 \%$ pulse mode. The product was filtered and washed with concentrated $\mathrm{HCl}$ to remove $\mathrm{MnO}_{2}$ [8].

The polyurethane membrane for MWCNT dispersion filtration was prepared by electrospinning from polyurethane dimethyl formamide/methyl isobutyl ketone (1:3) solution in cooperation with the SPUR a.s. company of the Czech Republic (detailed schematic of experimental set-up is illustrated in [9]).

To make an entangled MWCNT network, a porous polyurethane membrane (Fig. 1a) and a vacuum filtration method was used. The homogenized dispersion of MWCNTs was vacuum filtered through the membrane. The filtered MWCNT network (thickness about $35 \mu \mathrm{m}$ ) was washed several times with deionized water and methanol in situ and dried between two glass micro fibers filter papers at $40^{\circ} \mathrm{C}$ for 24 hours. The partial infiltration of MWCNTs into the filter pores creates interlocking MWCNTs with the membrane 
which strengthens when the porous filter is transformed into the polymeric film during the compression molding at $175^{\circ} \mathrm{C}$. The initial state of infiltration is shown in Fig. 1b. The crosssection of the composite, after compression molding, is shown in Fig. 1c. The interaction between MWCNTs and the porous filter is mechanical before compression molding. However, the molten polymer apparently encloses MWCNTs from the surface during the molding at $175{ }^{\circ} \mathrm{C}$, making firm connection between them. We assume that this firm connection between layers sustains composite conductance even at extreme elongation and the reversibility of deformation and electrical conductance as well.

\section{RESULTS}

\section{A. Compression Detection}

The interconnected CNT structures are capable of detection of macroscopic electrical resistance change induced by their deformation. The published data show that the electrical response to strain or stress is sufficient and the sensing can be performed in real time [2,3,10-13].

To show the compression sensing abilities of the composite in practice, we measured the pressure between a shoe sole and the ground while a volunteer marched in place. A strip of MWCNT/PU composite was glued to a shoe sole. The electrical contacts were fixed to the strip by a silver-colloid electro-conductive paint Dotite D-550 (SPI Supplies) and the resistance was measured lengthwise by the two-point technique using a Sefram 7338 multimeter. The time-dependent resistance change was monitored through the Vernier LabQuest Interface System connected to a differential voltage probe and the Wheatstone bridge with sampling frequency 100 $\mathrm{Hz}$.

The recorded waveform in terms of normalized resistance $\Delta R / R_{0}=\left(R-R_{0}\right) / R_{0}$, where $R_{0}$ is the electrical resistance of the measured sample before the first compression and $R$ is the resistance while compressed, is shown in Fig. 2. It is apparent that the resistance of the sensor is reversible not only in the five cycles displayed above, but also over a long period, as illustrated by Fig. 3. The stepping frequency was about $1 \mathrm{~Hz}$, which resulted in 90 cycles during which, as can be seen in Fig. 3, the sensor resistance is oscillating between two almost constant values.

\section{B. Deformation by Bending}

The change of electrical resistance of the composite during the static and dynamic bending combines the response to compression and stretching. The smaller the bend radius, the higher composite strip deformation and consequently, the higher resistance change, Fig. 4.

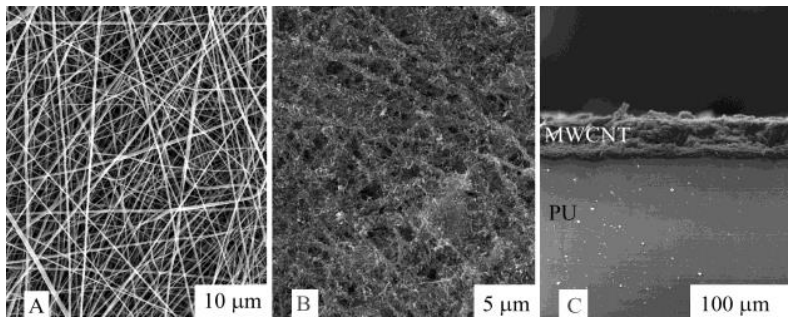

Fig. 1. A) SEM micrograph of polyurethane non-woven filter membrane, B) the filtering membrane at the initial stage of filtration process with entrapped nanotubes, C) the composite cross-section after the compression molding.

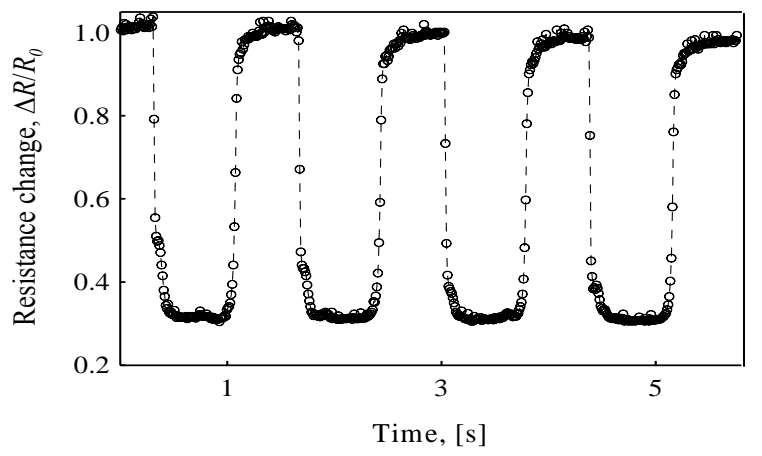

Fig. 2. Waveform of the normalized resistance during stepping detected by MWCNT/PU sensor adhered to the sole of a shoe (solid symbols).

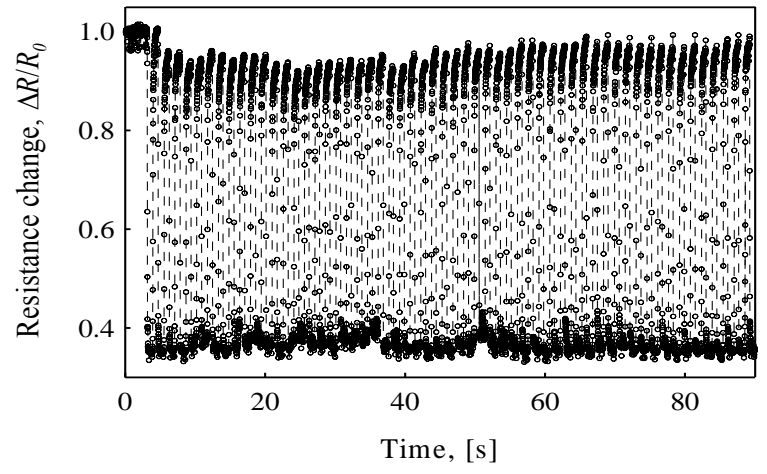

Fig. 3. The normalized resistance cycles in the course of plantar pressure measurement, showing the sensor's repetitive properties.

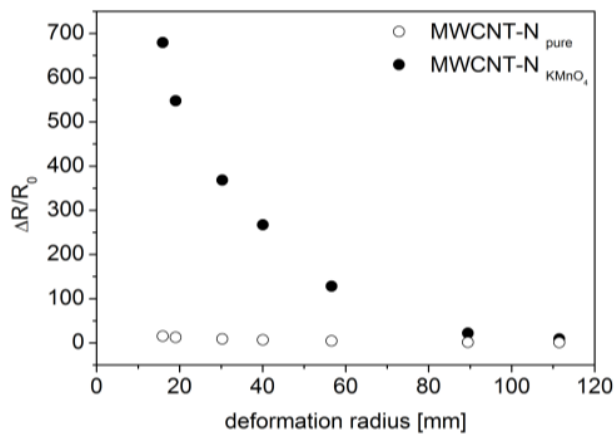

Fig. 4. The relative resistance change vs. bending radius of the mesured specimen with pristine Baytubes C70 MWCNTs (open circles) and $\mathrm{KMnO}_{4}$ MWCNTs (solid circles). 
The resistance change of the composite with pristine MWCNTs (BT) and oxidized MWCNTs (BT) was compared at the radius $40 \mathrm{~mm}$. The change for pristine MWCNTs is about $6.76 \%$ and for the oxidised MWCNTs about $267 \%$. The corresponding gauge factor for pristine MWCNTs is $G F=4.50$, and for oxidized MWCNTs $G F=178$. The deformation $\varepsilon$ of the composite strip was approximately $15.5 \%$.

The waveform in Fig. 5 shows how fast the time dependent response of the strip of the cantilevered composite is to a sudden initial deflection of the unattached end. The measurement demonstrates the composite measuring properties during high frequency deformation.

\section{Sensing of Tensile Deformation}

The data presented in Fig. 6 show that MWCNT/PU is electrically conductive even at the highest strain of about 400 $\%$ when the resistance is more than $47000 \Omega\left(\Delta \mathrm{R} / \mathrm{R}_{0}=277\right)$. The resistance increase under strain is continuous without any discontinuity, which is not usual in the case of conductive particulate composites, when an electrical percolation threshold is reached at greater strain. Moreover, the resistance of the maximally elongated composite returns in the unloaded state to the residual value $1750 \Omega\left(\Delta R / R_{0}=10.29\right)$ which differs from the initial resistance value of $170 \Omega$. The residual strain of the composite in the unloaded state is $24.7 \%$ due to the composite irreversible deformation in the first highly deforming cycle [3]. The gauge factor for the elongating composite can be calculated using data from Fig. 6 . The gauge factor increases with strain values around 4 at the beginning of deformation to nearly 69 at a strain $400 \%$. This is a substantial increase, which puts MWCNT/PU composite in the range of composite materials and strain gauges with the highest sensitivity to tensile deformation.

Fig. 7 shows waveforms of the test performed with the measuring strip of MWCNT/PU composite on a knee bandage pedaling more than $16 \mathrm{~min}$ on an exercise bicycle to demonstrate the composite measuring properties under longterm cyclic stretching. A pedaling frequency about $1 \mathrm{~Hz}$ resulted more than 1000 cycles during which the composite resistance changes were repetitive and no material property variation is observed. Though a commercial sensor made from MWCNT network/PU composite should withstand multiples of test cycles, we believe that the composite has reasonable durability and reversibility of the base characteristics for practical application [3].

\section{Sensing of Organic Vapors}

CNT network structure is suitable for its use as a membrane [14], adsorbent [15] or gas sensor [16,17]. Gas and vapor adsorption as well as desorption usually proceeds at high rates and amounts [18]. The data in Fig. 8 represents the change of resistance MWCNT(BT)/PU composite cyclically exposed to saturated vapors of ethanol and heptane in

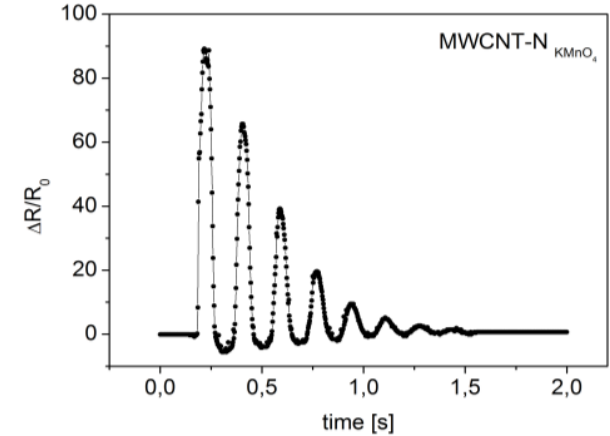

Fig. 5. Time-dependent relative resistance change response of the cantilevered composite strip to a rapid deflection of the loose end.

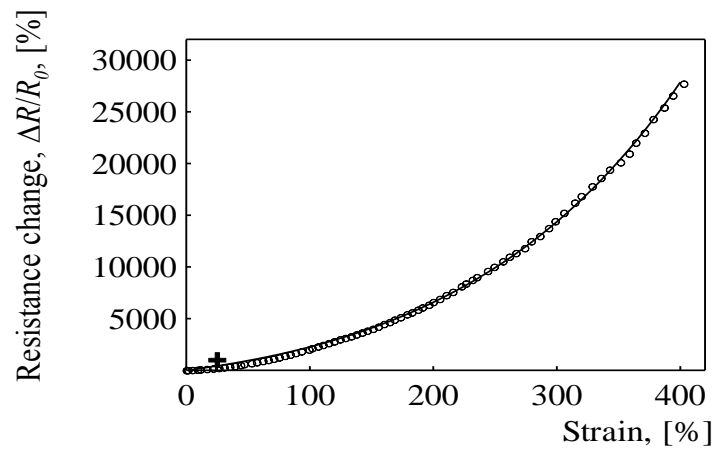

Fig. 6. Strain dependence of relative resistance change of MWCNT/PU composite. The cross denotes the resistance change value in a relaxed state after $400 \%$ extension [3].

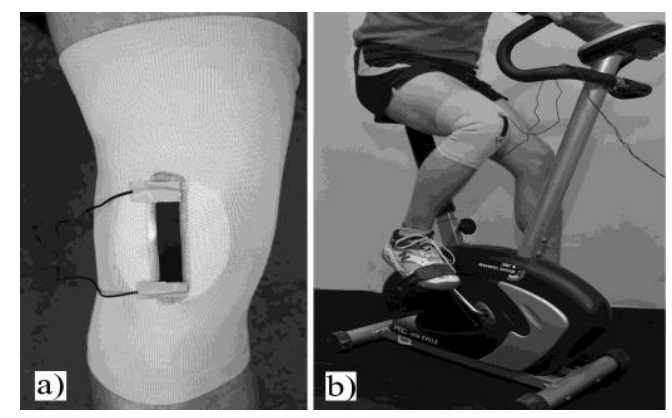

c)

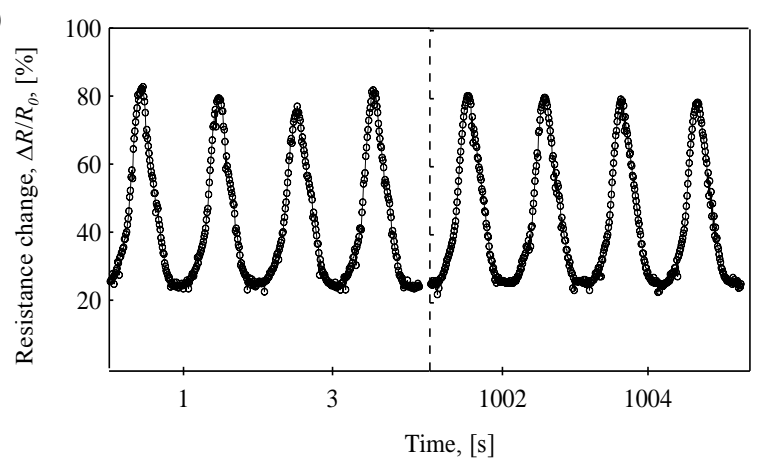

Fig. 7. a) Position of measured strip of MWCNT/PU composite on the knee bandage. b) Exercise bicycle setup for the measurement of cyclical deformation. c) The waveform of the normalized resistance during cycling [3]. 


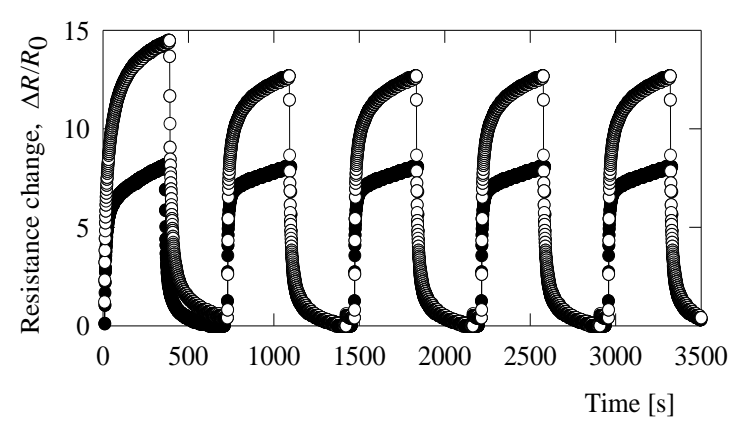

Fig. 8. Five adsorption/desorption cycles for MWCNT(BT)/PU composite exposed to vapors of ethanol (open circles) and heptane (solid circles).

adsorption/desorption cycles. The solvents were chosen to have similar values of saturated vapor pressure, $p_{i}$, which defined corresponding volume fractions of vapors in air, $x_{i}$, with considerably different polarities defined by Hildebrand solubility parameter, $\delta_{t}$. Calculated values of $x_{i}$ are 7.8 vol. \% for ethanol and $6.0 \mathrm{vol} . \%$ for heptane at $25^{\circ} \mathrm{C}$ with $\delta_{t}=26$ $\mathrm{MPa}^{1 / 2}$ and $\delta_{t}=15 \mathrm{MPa}^{1 / 2}$ for ethanol and heptane, respectively.

The adsorption of vapor molecules causes increase in the resistance and desorption of vapor molecules causes decrease of macroscopic resistance when non-conductive layers between the tubes is desorbed [19,20]. The curves in Fig. 8 have specific shapes of adsorption/desorption, with a clear on/off effect. An initial sharp increase in resistance is followed by a slower phase. Experimental data demonstrates also good reversibility of adsorption/desorption processes. The increase in resistance in the present of polar ethanol compared to the non-polar heptane is higher. This is probably caused by a greater affinity of ethanol to the MWCNTs.

\section{CONCLUSIONS}

We have introduced a highly deformable composite composed of a network of electrically-conductive entangled carbon nanotubes embedded in elastic polyurethane. The composite was prepared by taking a polyurethane filter membrane, enmeshing it with carbon nanotubes and melting them into one piece. This procedure eliminates the laborious process of peeling the nanotube network from the common micro-porous (polycarbonate, nylon) filter, followed by impregnation that increases its compactness. Testing has shown the composite can be compressed, bent, extended and exposed to chemical vapors to cause corresponding change of its resistance. This straightforward relationship indicates the possible use of this composite as a multifunction sensor.

\section{ACKNOWLEDGMENT}

The work was supported by the European Regional Development Fund, the Centre of Polymer Systems project (Reg. No.: CZ.1.05/2.1.00/03.0111), the grant of TBU in Zlin No. IGA/FT/2012/022 and by the Fund of the Institute of Hydrodynamics AV0Z20600510.

\section{REFERENCES}

[1] S. M. Vemuru, R. Wahi, S. Nagarajaiah, P. M. Ajayan, "Strain sensing using a multiwalled carbon nanotube film," J. Strain Anal. Eng. Des., vol. 44, pp. 555-562, 2009.

[2] I. P. Kang, M. J. Schulz, J. H. Kim, V. Shanov, D. L. Shi, “ A carbon nanotube strain sensor for structural health monitoring," Smart Mater. Struct., vol. 15, pp. 737-748, 2006.

[3] P. Slobodian, P. Riha, P. Saha, "A highly-deformable composite composed of an entangled network of electrically-conductive carbonnanotubes embedded in elastic polyurethane," Carbon, vol. 50, pp. 3446-3453, 2012.

[4] P. Slobodian, P. Riha, R. Olejnik, "Electromechanical sensors based on carbon nanotube networks and their polymer composites. In: New Developments and Applications in Sensing Technology, S. C. Mukhopadhyay, A. Lay-Ekuakille, A. Fuchs, Eds. , Springer Verlag, 2011, p. 233-251.

[5] A. De la Vega, I. A. Kinloch, R. J. Young, W. Bauhofer, "Simultaneous global and local strain sensing in SWCNT-epoxy composites by Raman and impedance spectroscopy," Compos. Sci. Technol., vol. 71, pp. 160$166,2011$.

[6] E. Bilotti, R. Zhang, H. Deng, M. Baxendale, T. Peijs, "Fabrication and property prediction of conductive and strain sensing TPU/CNT nanocomposite fibres," J. Mat. Chem., vol. 20, pp. 9449-9455, 2010.

[7] P. Slobodian, P. Riha, A. Lengalova, P. Saha, "Compressive stresselectrical conductivity characteristics of multiwall carbon nanotube networks," J. Mater. Sci., vol. 46, pp. 3186-3190. 2011.

[8] P. Slobodian, P. Riha, A. Lengalova P. Svoboda, P. Saha, "Multi-wall carbon nanotube networks as potential resistive gas sensors for organic vapor detection," Carbon, vol. 49, pp. 2499-2507, 2011.

[9] D. Kimmer, P. Slobodian, D. Petras, M. Zatloukal, R. Olejnik, P. Saha, "Polyurethane/multiwalled carbon nanotube nanowebs prepared by an electrospinning process," J. Appl. Polym. Sci., vol. 111, pp. 2711-2714, 2009.

[10] E. T. Thostenson, T. W. Chou, "Real-time in situ sensing of damage evolution in advanced fiber composites using carbon nanotube networks," Nanotechnology, vol. 19, article no. 215713, 2008.

[11] M. D. Rein, O. Breuer, H. D. Wagner, "Carbon nanotube buckypaper films as strain sensing devices," Compos. Sci. Technol., vol. 71, pp. 373-381, 2011.

[12] G. T. Pham, Y. B. Park, Z. Liang, C. Zhang, B. Wang, "Processing and modeling of conductive thermoplastic/carbon nanotube films for strain sensing," Compos. Pt. B-Eng., vol. 39, pp. 209-216, 2008.

[13] P. Slobodian, P. Riha, A. Lengalova, R. Olejnik, P. Saha, "Effect of compressive strain on electric resistance of multi-wall carbon nanotube networks," J. Exp. Nanosci., vol. 6, pp. 294-304, 2011.

[14] R. Smajda, A. Kukovecz, Z. Konya, I. Kiricsi, "Structure and gas permeability of multi-wall carbon nanotube buckypapers.," Carbon, vol. 45, pp. 1176-1184, 2007.

[15] S. Agnihotri, J. P. B. Motam, M. Rostam-Abadi, M. J. Rood, "Theoretical and experimental investigation of morphology and temperature effects on adsorption of organic vapors in single-walled carbon nanotubes," J. Phys. Chem. B, vol. 110, pp. 7640-7647, 2006.

[16] A. I. Romanenko, O. B. Anikeeva, V. L. Kuznetsov, T. I. Buryakov, E. N. Tkachev, A. N. Usoltseva, "Influence of helium, hydrogen, oxygen, air and methane on conductivity of multiwalled carbon nanotubes," Sensor Actuat. A-Phys., vol. 138, pp. 350-354, 2007.

[17] A. Qureshi, W. P. Kang, J. L. Davidson, Y. Gurbuz, "Review on carbonderived, solid-state, micro and nano sensors for electrochemical sensing application," Diam. Relat. Mater., vol. 18, pp. 1401-1420, 2009.

[18] C. M. Hussain, C. Saridara, S. Mitra, "Microtrapping characteristics of single and multi-walled carbon nanotubes," J. Chromatogr. A, vol. 1185, pp. 161-166, 2008.

[19] F. Tournus, S. Latil, M. I. Heggie, J. C. Charlier, "Pi-stacking interaction between carbon nanotubes and organic molecules," Phys. Rev. B, vol. 72, article no. 075431, 2005.

[20] D. J. Mowbray, C. Morgan, K. S. Thygesen, "Influence of O-2 and N-2 on the conductivity of carbon nanotube networks," Phys. Rev. B, vol. 79, article no. 195431, 2009. 